Barbara CALLAWAY et Lucy CREEVEY, The Heritage of Islam. Women, Religion and Politics in West Africa, Boulder et Londres, Lynne Rienner Publishers, 1994, $221 \mathrm{p}$.

Jean-Louis TRIAUD

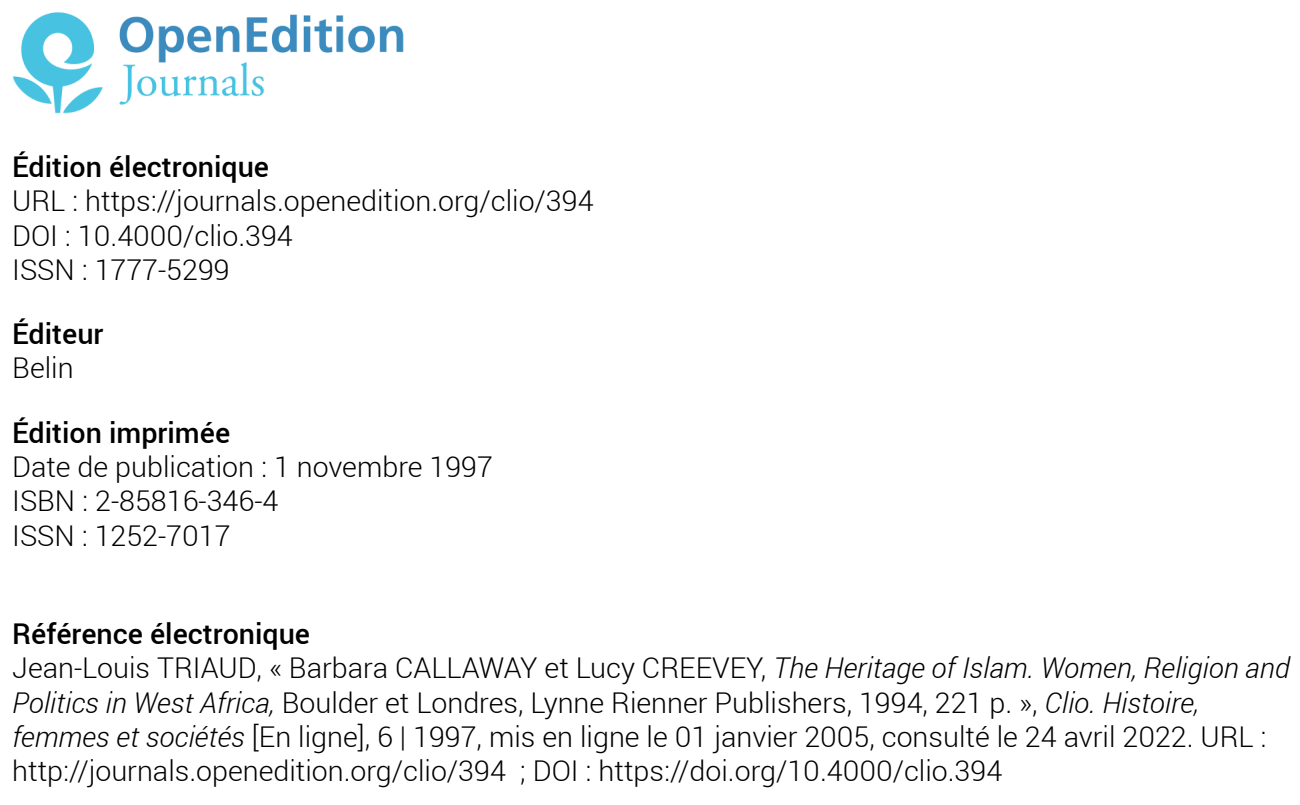

Ce document a été généré automatiquement le 24 avril 2022.

Tous droits réservés 


\title{
Barbara CALLAWAY et Lucy CREEVEY, The Heritage of Islam. Women, Religion and Politics in West Africa, Boulder et Londres, Lynne Rienner Publishers, 1994, 221 p.
}

\author{
Jean-Louis TRIAUD
}

1 Les travaux sur le rôle et la place des femmes en Afrique subsaharienne ne sont pas très nombreux. On pense notamment pour les pionniers/ères à la thèse de Catherine Coles, Muslim Women in Town. Social Change among the Hausa of Northern Nigeria (Madison, Ph. D, 1983), à Jean Boyd et Murray Last, "The Role of Women as "Agents religieux " in Sokoto » (Canadian Journal of African Studies, 1985), à Barbara Callaway, Muslim Hausa Women in Nigeria. Tradition and Change (Syracuse, 1987), à Jean Boyd, The Caliph's Sister, Nana Asma'u, 1793-1865: Teacher, Poet and Islamic Leader (Londres, 1989), à Christian Coulon, « Women, Islam and Baraka », dans Coulon et Cruise O'Brien (eds), Charisma and Brotherhood in African Islam (Oxford, 1989), à Coles et Mack (eds), Women in TwentiethCentury Hausa Society, qui contient plusieurs communications importantes sur les rapports entre femmes et religion (Madison, 1991), ainsi qu'à une publication commune antérieure de Callaway et Creevey, "Women and the State in Islamic Africa», dans Charlton, Everett et Staudt (eds), Women, the State and Development (Albany, 1989). Depuis le début des années 1990, ce nouveau courant de publications sur les femmes et l'islam en Afrique de l'Ouest semble s'être ralenti. Cette réserve générale tient autant à une tradition «masculine » de la recherche islamisante qu'au caractère «patriarcal » de nombre de sociétés africaines, notamment islamiques.

2 À travers cette enquête, les auteurs veulent montrer que " a plupart des sociétés, aussi restrictives soient-elles, ont une structure de pouvoir féminine dans l'ombre de la structure masculine publique». Et, s'agissant des confréries, "regarder de plus près peut permettre de découvrir la structure "dans l'ombre " à l'intérieur » de celles-ci 
(pp. 44-45). Sur la base d'une distinction entre "pouvoir» (qui implique la décision) et « influence» (qui ne la permet pas), les auteurs précisent en ces termes, dans leur introduction, l'un des objectifs de leur recherche : « comment les femmes musulmanes dans la société ouest-africaine utilisent leur influence pour acquérir du pouvoir » (p. 4).

Barbara Callaway est une spécialiste de science politique, auteur de l'ouvrage cité cidessus sur les femmes musulmanes hausa au Nigeria (1987). Lucy Creevey (Behrman), également spécialiste de science politique, professeur à l'université du Connecticut, est l'auteur du premier ouvrage important sur les confréries sénégalaises, Muslim Brotherhoods and Politics in Senegal (Cambridge, E.-U., 1970) et l'éditeur d'un livre collectif sur les paysannes au Mali et dans les pays du Sahel (1986). Ainsi une spécialiste du Nigeria et une du Sénégal ont-elles combiné leurs compétences dans un ouvrage essentiellement comparatif qui place en vis-à-vis l'analyse des situations dans ces deux pays. Chaque chapitre, après une présentation générale, est composé, de façon systématique, de deux parties relatives au Nigeria, puis au Sénégal les deux "poids lourds » en matière d'islam ouest-africain, même si le rapport numérique est fort inégal (50 millions de musulmans dans le premier cas, autour de 7 millions dans le second). L'exposé est découpé en quatre chapitres: historique, sociologique, économique et politique, où sont envisagés le rôle et la place des femmes dans ces différentes instances. Les auteurs s'attachent à montrer que l'islam n'est pas toujours le facteur essentiel, ni même dominant, de la mise en dépendance des femmes, et qu'il convient d'analyser concrètement les différentes situations. Ainsi, dans le domaine politique, « les femmes dans une société musulmane avec un État séculier où les fondamentalistes sont faibles ne semblent pas se heurter à des barrières à leur avancement à cause de leur religion. Par contre, là où l'islam orthodoxe est fort et où les Musulmans constituent une puissante minorité, le gouvernement doit faire attention à ne pas mettre en cause la Shari'a de quelque manière que ce soit. Ce qui est important n'est pas de savoir simplement si une femme ouest-africaine est, ou non, musulmane, mais comment le système politique de sa société est structuré et quel pouvoir ou influence exerce sa religion » (p. 186). La comparaison entre les deux pays met au jour en effet les effets contrastés d'un héritage " théocratique » dans le cas nigerian, qui s'accompagne de la réclusion de nombre de femmes et de la tendance au port du voile, et d'une tradition séculière libérale dans le cas sénégalais, qui tient à une longue familiarité avec le modèle français, et qui, si l'on y ajoute un héritage matrilinéaire puissant, n'entraîne ni port du voile ni réclusion pour les femmes. Est-ce à dire que cette enquête conclut, de façon banale, aux vertus de la laïcité à la française et de l'occidentalisation? Les deux auteurs ne le pensent pas. Montrant les effets induits à long terme de la nouvelle sociabilité féminine islamique, elles insistent sur les processus en cours au Nigeria: "Même si les femmes musulmanes plus observantes du Nigeria paraissent, au premier abord, vivre des vies plus limitées que leurs soeurs sénégalaises, elles peuvent en fait être à l'avant-garde d'une réinterprétation de l'islam susceptible d'avoir des conséquences révolutionnaires dans leur propre société. Elles mettent celle-ci en question afin de mieux vivre en accord avec les principes de leur religion et, ce faisant, elles montrent le chemin de réinterprétations de la doctrine islamique qui peuvent inaugurer une nouvelle tradition islamique au Nigeria et influencer également l'islam à travers l'Afrique de l'Ouest » (p. 196). Tels sont les derniers mots de cet ouvrage qui a le grand mérite de rassembler et de comparer des données utiles sur deux pays rarement 
traités conjointement en raison de leur appartenance respective à l'Afrique de l'Ouest anglophone et francophone.

4 On regrettera quelques coquilles dans l'exposé historique : il faut lire Al-Maghîlî et non «al-Maghrib» (p. 20) ; al-Hajj Umar est mort en 1864, et non en 1861 (p. 21). La présentation du passé islamique " toucouleur » est un peu limitée : il aurait été utile de rappeler l'importance de la révolution islamique qui a eu lieu dans la vallée du Sénégal à la fin du XVIII ${ }^{\text {ème }}$ siècle et qui offre un élément de comparaison avec le jihâd de Sokoto, au Nigeria. Seul le jihâd d'al-Hajj Umar est connu (p. 20). Le chapitre se conclut par la mention curieuse des «Islamic and French invasions of Senegal » qui auraient combiné leurs effets pour amoindrir le statut des femmes (p. 27). Quelle est donc cette « invasion islamique » mise en équivalence avec celle des Français étant entendu que la diffusion de l'islam au Sénégal n'est pas le résultat d'une invasion arabe ou étrangère, mais le produit d'un processus au long cours?

Il faut voir ce livre comme un essai de bilan comparé dans deux pays qui tiennent une place majeure dans l'évolution de l'islam ouest-africain, et comme une contribution, en termes positifs et optimistes, à la compréhension des changements en cours dans les rôles sociaux des femmes ouest-africaines. 